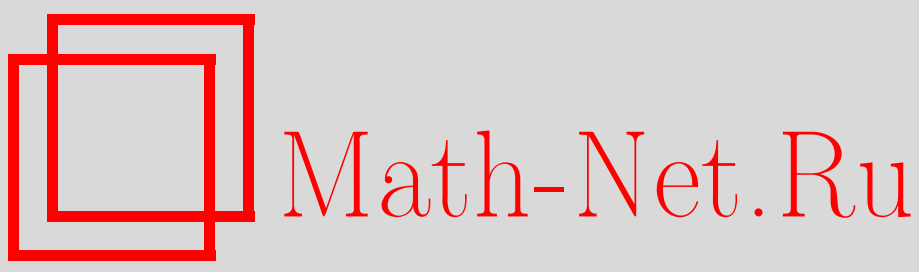

Е. Е. Тыртышников, Тензорные аппроксимации матриц, порожденных асимптотически гладкими функциями, Матем. сб., 2003, том 194, номер 6, 147-160

DOI: https://doi.org/10.4213/sm747

Использование Общероссийского математического портала Math-Net.Ru подразумевает, что вы прочитали и согласны с пользовательским соглашением

http://www . mathnet.ru/rus/agreement

Параметры загрузки:

IP: 54.197 .217 .227

26 апреля 2023 г., 14:10:08 
УДК $512.64+517.5$

\author{
Е.Е. Тыртышников
}

\title{
Тензорные аппроксимации матриц, порожденных асимптотически гладкими функциями
}

\begin{abstract}
Для шшрокого класса матриц (дискретных аналогов типичных интегральных операторов) установлена возможность аппроксимации суммой прямых произведений матриц меншего размера. Получены оценки числа членов (тензорного ранга) и соответствуюшей погрешности. Показано, что тензорные аппроксимации как метод сжатия данњшх обеспечивают сверхлинейное сжатие.

Библиография: 19 названий.
\end{abstract}

\section{§1. Введение}

Во многих прикладных задачах (например, при дискретизации интегральных уравнений) возникает необходимость решать линейные системы $(A x=f)$ с большими плотньми матрицами. Классические алгоритмы исключения элементов требуют огромных вычислительных затрат и поэтому малополезны. В отдельных случаях, когда плотная матрица имеет специальный вид (например, для тёплицевых матриц) удается построить специальные алгоритмы малой вычислительной сложности [1], [2]. Однако в общем случае остается, по сути, только один путь - аппроксимировать $A$ "хорошей" матрицей специального вида $A_{r}$ и строить эффективные алгоритмы решения для $A_{r}$. При использовании быстро сходящихся итерационных алгоритмов (например, метода сопряженных градиентов и его обобщений) можно ограничиться задачей быстрого умножения матрицы $A_{r}$ на вектор. В большинстве известных подходов для этой цели явным образом используют вид ядра интегрального оператора [3]-[6]. Матричная трактовка методов обсуждается в [7]-[11]. Методы аппроксимации, использующие только элементы исходной матрицы, развиваются в работах [12]-[16].

В упомянутых вьше работах матрица $A_{r}$ является многоуровневой блочной матрицей с блоками, ранг которых мал по сравнению с порядком исходной матрицы. Таким образом, блоки представляются суммами матрищ вида $u v^{T}$, где $u$ и $v$-вектор-столбцы (скелетоны). При определенных предположениях общее число компонент этих скелетонов имеет вид $O\left(n \log ^{\gamma} n\right)$, где $n$-порядок матрицы $A$, а $\gamma$ не зависит от $n$.

Соответствуюшие методы аппроксимации сводятся к построению многоуровневого блочного разбиения матрицы с определенной иерархией блоков и “локальной” скелетонной аппроксимации каждого отдельного блока. В настояшей работе мы рассмотрим подход, в котором нет логически сложной конструкции разбиений

Работа вьполнена при поддержке Российского фонда фундаментальных исследований (грант № 02-01-00590) и Фонда поддержки отечественной науки.

(C) Е.Е. Тыртышников 2003 
на блоки и аппроксимации матрицы имеют “глобальный" характер. Это будут тензорные аппроксимации следующего вида:

$$
A \approx A_{r}=\sum_{k=1}^{r} V_{k}^{1} \times \cdots \times V_{k}^{m}
$$

Символ “ $\times$ " обозначает прямое (кронекерово) произведение матриц: если $P=$ $\left[p_{i j}\right]$ имеет размер $m_{P} \times n_{P}$ и $Q$ имеет размер $m_{Q} \times n_{Q}$, то прямое произведение

$$
P \times Q=\left[p_{i j} Q\right]
$$

есть матрица размера $\left(m_{P} m_{Q}\right) \times\left(n_{P} n_{Q}\right)$. Таким образом, произведение строчных (соответственно столбцовых) размеров матриц $V_{k}^{1}, \ldots, V_{k}^{m}$ из (1) равно порядку матрицы $A$. Будем считать, что матрица $V_{k}^{l}$ имеет размер $n_{l} \times n_{l}$. Значит,

$$
n=n_{1} \cdots n_{m}
$$

Чтобы объяснить пользу тензорных аппроксимаций для вычислений, предположим, что $n_{1}=\cdots=n_{m}=p$. Тогда общее число элементов матрицы $A$ равно $p^{2 m}=n^{2}$, а общее число элементов в матрицах $V_{k}^{l}$ равно $r m p^{2}=r m n^{2 / m}$. Таким образом, при фиксированных $m$ и $r$ объем памяти для хранения тензорной аппроксимации $A_{r}$ составляет $m r n^{2 / m-2}$ - лишш малую часть от $n^{2}$ - объема памяти для хранения исходной матрицы $A$. При умножении $A_{r}$ на вектор требуется лишш порядка $r m p^{m-1} p^{2}=r m n^{1+1 / m}$ арифметических операций - в отличие от $n^{2}$ операций для исходной матрицы $A$.

Обратим внимание на то, что при $m \geqslant 3$ тензорные аппроксимации как метод сжатия данных превосходят любые варианты упомянутых выше блочных скелетонных методов. Они дают сверхлинейное сәсатие: отношение объема требуемой памяти для хранения аппроксимации к объему исходной памяти $\left(n^{2}\right)$ есть величина порядка $n^{2 / m-2}$ и в случае $m \geqslant 3$ при $n \rightarrow \infty$ стремится к нулю быстрее, чем $1 / n$.

В работе [17] замечено, что с практической точки зрения тензорные представления интересны даже в случае, когда $A$ является многоуровневой тёплицевой матрицей, а значит, для ее умножения на вектор можно использовать алгоритм быстрого преобразования Фурье сложности $O(n \log n)$. Пусть $r=1, m=3$ и $p=2^{s}$. Тогда (в случае комплексных матрици векторов) для умножения трехуровневой тёплицевой матрицы порядка $n$ на вектор (на основе алгоритма Кули-Тьюки) достаточно вьполнить в главном члене $[2 ; \S 4$, с. 89]

$$
3 \cdot\left(1+\frac{2^{m}-1}{m 2^{m}}\right) \cdot 2 n \log _{2} n=\frac{279}{12} n s
$$

комплексных операций, а при использовании тензорного представления достаточHO

$$
2 r m n^{1+1 / m}=6 n 2^{s / 3}
$$

операций. Неравенство

$$
\frac{279}{72} s \leqslant 2^{s / 3}
$$


начинает выполняться при $s \geqslant 19$. Таким образом, тензорное представление выгоднее при $n<2^{57}$ (хотя и проигрывает асимптотически).

В работе [18] впервые даны описание широкого класса матриц и доказательство того, что для них существуют достаточно точные тензорные аппроксимации с малым рангом $r$. Проведенные в этой работе построения ограничиваются, однако, случаем $m=2$ и предположением о том, что элементы матрицы $A$ представляют собой значения некоторой асимптотически гладкой функции на прямоугольной сетке в квадрате на плоскости. Сделанное выше замечание показьвает особый интерес к тензорным аппроксимациям с $m \geqslant 3$, а в приложениях наибольшие трудности связаны с сетками в трехмерном пространстве и в пространствах большей размерности. Цель настоящей работы - представить результаты о существовании "малоранговых" тензорных аппроксимаций в этих более сложных случаях.

\section{§ 2. Прямые произведения и скелетоны}

Пусть $A=V^{1} \times \cdots \times V^{m}$, где $V^{l}$ - матрица порядка $n_{l}, 1 \leqslant l \leqslant m$. Такую матрицу $A$ естественно рассматривать как $m$-уровневую с уровнями порядков $n_{1}, \ldots, n_{m}$ [2]. Это означает, что $A$ является блочной $\left(n_{1} \times n_{1}\right)$-матрицей, в которой каждый блок есть блочная $\left(n_{2} \times n_{2}\right)$-матрица и т. д. Порядок матрицы $A$ равен $n=n_{1} \cdots n_{m}$. При указании позиции элемента $a_{i j}, 1 \leqslant i, j \leqslant n$, матрицы $A$ удобно использовать мультииндексы

$$
i \leftrightarrow \mathbf{i}=\left(i_{1}, \ldots, i_{m}\right), \quad j \leftrightarrow \mathbf{j}=\left(j_{1}, \ldots, j_{m}\right)
$$

при этом выполняются следующие соотношения:

$$
\begin{gathered}
i=\left(i_{1}-1\right) n_{2} \cdots n_{m}+\left(i_{2}-1\right) n_{3} \cdots n_{m}+\cdots+\left(i_{m-1}-1\right) n_{m}+i_{m} \\
j=\left(j_{1}-1\right) n_{2} \cdots n_{m}+\left(j_{2}-1\right) n_{3} \cdots n_{m}+\cdots+\left(j_{m-1}-1\right) n_{m}+j_{m} \\
1 \leqslant i_{l}, j_{l} \leqslant n_{l}, \quad 1 \leqslant l \leqslant m .
\end{gathered}
$$

Многоуровневую структуру можно вводить также для прямоугольных матриц и разного числа уровней для строк и столбцов. В таких случаях строчные и столбцовые мультииндексы имеют разное число компонент.

Матрица $A$ естественным образом порождает, в частности, такие матрицы:

$$
\begin{gathered}
A_{l}=\left[a_{\mathbf{p q}}^{l}\right], \quad \mathbf{p}=\left(i_{1}, j_{1}, \ldots, i_{l-1}, j_{l-1}\right), \quad \mathbf{q}=\left(i_{l}, j_{l}, \ldots, i_{m}, j_{m}\right), \\
a_{\mathbf{p q}}^{l}=a_{\mathbf{i j}} .
\end{gathered}
$$

Заметим, что $A_{l}$ имеет размер $\left(n_{1}^{2} \cdots n_{l-1}^{2}\right) \times\left(n_{l}^{2} \cdots n_{m}^{2}\right)$.

ПрЕДЛОЖЕНИЕ 1. Для того чтобы матрица А была суммой не более чем $r$ прямых произведений $m$ матриц порядков $n_{1}, \ldots, n_{m}$, необходимо, чтобь кажсдая из матрии, $A_{l}$ имела ранг не выше $r$.

В случае $m=2$ аналогичное наблюдение было сделано в [19], при этом условие на ранг является также достаточным. В этом случае предложение 1 позволяет строить тензорные аппроксимации минимального ранга с гарантированной 
точностью по норме Фробениуса (корень квадратный из суммы квадратов модулей элементов) на основе скелетонного приближения для $A_{2}$, которое можно вычислить с помощью стандартного алгоритма сингулярного разложения.

В случае $m \geqslant 3$ предложение 1 позволяет найти нижнюю оценку для $r$ (с помощью сингулярного разложения матриц $A_{l}$ ). Оно же (путем рекурсивного применения) дает возможность построить некоторую тензорную аппроксимацию, но не обязательно наименышего возможного ранга при заданной точности.

В наших построениях возникают не только $m$-уровневые, но и $m$-мерные матрицы. Под $m$-мерной матрицей $B$ понимается таблица, в которой элементы позиционируются с помощью $m$ индексов, или дискретная функция $b_{\alpha_{1}, \ldots, \alpha_{m}}$ на декартовом произведении $m$ индексных множеств. Будем писать $B=\left[b_{\alpha_{1}}, \ldots, \alpha_{m}\right]$. Индексные множества многомерной матрицы могут иметь многоуровневое строение, в этом случае мы используем мультииндексы.

Ассоциируем с матрицей $A=V^{1} \times \cdots \times V^{m}$ следующую $m$-мерную матрицу:

$$
A \leftrightarrow \mathscr{P}(A)=\left[b_{\mathbf{p}_{1}, \ldots, \mathbf{p}_{m}}\right], \quad \mathbf{p}_{1}=\left(i_{1}, j_{1}\right), \ldots, \mathbf{p}_{m}=\left(i_{m}, j_{m}\right)
$$

ПРЕДЛОЖЕНИЕ 2. Для того чтобъ матрица А бъла суммой $r$ прямых произведений $m$ матрии порядков $n_{1}, \ldots, n_{m}$, необходимо и достаточно, чтобъ элементы матрииьи $\mathscr{P}(A)$ имели вид

$$
b_{\mathbf{p}_{1}, \ldots, \mathbf{p}_{m}}=\sum_{k=1}^{r} u_{\mathbf{p}_{1}}^{k} \cdots u_{\mathbf{p}_{m}}^{k}
$$

для каких-то значений $u_{\mathbf{p}_{1}}^{k}, \ldots, u_{\mathbf{p}_{m}}^{k}$.

Если $r$ - наименьшее возможное число слагаемых, то будем говорить, что $\mathscr{P}(A)$ есть $m$-мерная матрица тензорного ранга $r$, представленная в виде суммы $m$-мерных скелетонов (двумерньй скелетон - это обычная (двумерная) матрица ранга 1).

\section{§3. Сепарабельные аппроксимации}

Предположим, что

$$
a_{i j}=f\left(\mathbf{x}^{i}, \mathbf{y}^{j}\right), \quad \mathbf{x}^{i}=\left(x_{1}^{i}, \ldots, x_{m}^{i}\right), \quad \mathbf{y}^{j}=\left(y_{1}^{j}, \ldots, y_{m}^{j}\right), \quad 1 \leqslant i, j \leqslant n
$$

где $f$ - заданная функция, а $\left\{\mathbf{x}^{i}\right\}$ и $\left\{\mathbf{y}^{j}\right\}$ - две системы точек (сетки) в $m$-мерном пространстве. Относительно функции и сеток необходимо сделать некоторые предположения.

Будем считать, что

$$
f(\mathbf{x}, \mathbf{y})=F(\mathbf{v}), \quad \mathbf{v}=\mathbf{x}-\mathbf{y}
$$

где $F$ дифференцируема всюду, кроме точки $\mathbf{v}=0$, и для смешанных производных

$$
D^{\mathbf{p}}=\frac{\partial^{p_{1}} \cdots \partial^{p_{m}}}{\left(\partial v_{1}\right)^{p_{1}} \cdots\left(\partial v_{m}\right)^{p_{m}}}, \quad \mathbf{p}=\left(p_{1}, \ldots, p_{m}\right)
$$


при любом $p=p_{1}+\cdots+p_{m} \geqslant 0$ имеют место следующие неравенства:

$$
\begin{gathered}
\left|D^{\mathbf{p}} F(\mathbf{v})\right| \leqslant c d^{p} p !\|\mathbf{v}\|^{g-p} \\
\mathbf{v}=\left(v_{1}, \ldots, v_{m}\right), \quad\|\mathbf{v}\|=\sqrt{v_{1}^{2}+\cdots+v_{m}^{2}}
\end{gathered}
$$

где $c, d>0$ и $g$ - константы, не зависящие от $\mathbf{p}$. Для определенности положим $F(0)=0$. Функцию $F$ с указанными свойствами будем называть nолной acuмnтотически гладкой.

Далее, рассмотрим системы одномерных сеток

$$
\begin{gathered}
0 \leqslant s_{1}^{l}<\cdots<s_{n_{l}}^{l} \leqslant 1, \quad 0 \leqslant t_{1}^{l}<\cdots<t_{n_{l}}^{l} \leqslant 1, \\
l=1, \ldots, m
\end{gathered}
$$

и будем считать, что

$$
\begin{array}{cl}
\mathbf{x}^{i}=\mathbf{x}^{\mathbf{i}}=\left(s_{i_{1}}^{1}, \ldots, s_{i_{m}}^{m}\right), & \mathbf{y}^{j}=\mathbf{y}^{\mathbf{j}}=\left(t_{j_{1}}^{1}, \ldots, t_{j_{m}}^{m}\right), \\
\mathbf{i}=\left(i_{1}, \ldots, i_{m}\right), & \mathbf{j}=\left(j_{1}, \ldots, j_{m}\right) .
\end{array}
$$

Определим минимальное расстояние между узлами сеток $\left\{\mathbf{x}^{i}\right\}$ и $\left\{\mathbf{y}^{j}\right\}$ следующим образом:

$$
h_{0}=\min _{1 \leqslant l \leqslant m} \min _{\substack{1 \leqslant \mu, \nu \leqslant n_{l} \\ \mu \neq \nu}}\left|s_{\mu}^{l}-t_{\nu}^{l}\right| .
$$

При $0<h<1$ будем рассматривать "прямоугольные кольца"

$$
\Pi_{h}=[-1,1]^{m} \backslash(-h, h)^{m} .
$$

При сделанных предположениях очевидно, что

$$
\mathbf{x}^{i}-\mathbf{y}^{j} \in \Pi_{h_{0}} .
$$

Полагая $\mathbf{v}=\left(v_{1}, \ldots, v_{m}\right)$, позволим себе писать $F(\mathbf{v})=F\left(v_{1}, \ldots, v_{m}\right)$. Чтобы изучить свойства тензорных аппроксимаций $A_{r} \approx A$, достаточно исследовать сепарабельные аппроксимации $F_{r} \approx F$, где

$$
F_{r}(\mathbf{v})=F_{r}\left(v_{1}, \ldots, v_{m}\right)=\sum_{k=1}^{r} \varphi_{k}^{1}\left(v_{1}\right) \cdots \varphi_{k}^{m}\left(v_{m}\right) .
$$

Главной целью сепарабельной аппроксимации является разделение переменных с гарантированной равномерной поточечной оценкой погрешности, поэтому в постановке задачи нет необходимости специфицировать класс функций, в котором следует искать $\varphi_{k}^{1}, \ldots, \varphi_{k}^{m}$.

ЛЕмма 1. Предположим, что для $F$ существует сепарабельная функиия $F_{r}$ вида (11) такая, что

$$
\left|F(\mathbf{v})-F_{r}(\mathbf{v})\right| \leqslant \varepsilon, \quad \mathbf{v} \in \Pi_{h_{0}} .
$$

Тогда для матрицы $A$ с әлементами $a_{i j}$ вида (3) существует тензорная аппроксимачия $A_{r}$ вида (1) с оценкой погрешности

$$
\left\|A-A_{r}\right\|_{C} \leqslant \varepsilon
$$

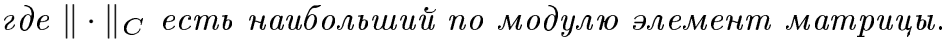

Мы опускаем доказательство, поскольку оно вполне очевидно.

В дальнейшем полагаем $\|\mathbf{v}\|^{g}=0$ в случае $\mathbf{v}=0$ (независимо от $g$ ). 
ТЕОРема 1. Предположим, что $F\left(v_{1}, \ldots, v_{m}\right)$ - полная асимптотически гладкая функиия, производные которой удовлетворяют неравенствам (5), и множество $\Pi_{h}$ имеет вид (9) при некотором $0<h<1$. Пусть $\gamma-$ произвольное число такое, что $0<\gamma<1$. Тогда для любого иелого $p \geqslant 0$ для $F$ существует сепарабельная аппроксимация $F_{r}$ вида (11) со следующими свойствами:

$$
\begin{gathered}
r \leqslant\left(c_{0}+c_{1} \log h^{-1}\right) p^{m-1} \\
\left|F(\mathbf{v})-F_{r}(\mathbf{v})\right| \leqslant c_{2} \gamma^{p}\|\mathbf{v}\|^{g}, \quad \mathbf{v} \in \Pi_{h},
\end{gathered}
$$

где константы $c_{0}, c_{1}, c_{2}$ зависят от $\gamma$ и $m$, но не зависят от $p$.

ДокАЗАТЕЛЬСтво. Покроем $\Pi_{h}$ семейством кубов $\left\{D_{\nu}\right\}$ (разных размеров) таким образом, что $F$ имеет на $D_{\nu}$ сепарабельную аппроксимацию с $p$ членами. Тогда очевидно, что $F$ будет обладать сепарабельной аппроксимацией на $\Pi_{h} \mathrm{c}$ $r \leqslant N p$ членами, где $N$ - общее число кубов.

Пусть $D$ - любой куб со стороной $a$, принадлежащий множеству

$$
\mathscr{R}(a)=[-(s+1) a,(s+1) a]^{m} \backslash(-s a, s a)^{m}
$$

где $s$ - целое положительное число, которое мы выберем позже.

Разложим $F$ в ряд Тейлора в центральной точке $\mathbf{v}^{0}=\left(v_{1}^{0}, \ldots, v_{m}^{0}\right)$ куба $D:$

$$
\begin{gathered}
F\left(v_{1}, \ldots, v_{m}\right) \approx F_{p}\left(v_{1}, \ldots, v_{m}\right) \\
=\left.\sum_{k=0}^{p-1} \frac{1}{k !}\left(\Delta v_{1} \frac{\partial}{\partial \xi_{1}}+\cdots+\Delta v_{m} \frac{\partial}{\partial \xi_{m}}\right)^{k} F\left(\xi_{1}, \ldots, \xi_{m}\right)\right|_{\xi_{1}=v_{1}^{0}, \ldots, \xi_{m}=v_{m}^{0}} \\
\Delta v_{1}=v_{1}-v_{1}^{0}, \ldots, \Delta v_{m}=v_{m}-v_{m}^{0} .
\end{gathered}
$$

Для оценки остаточного члена (в форме Лагранжа) воспользуемся неравенствами (5). При условии $\mathbf{v} \in D$ находим

$$
\left|F(\mathbf{v})-F_{p}(\mathbf{v})\right| \leqslant(p+1)^{m-1} c \frac{d^{p}}{(s a)^{p}}\left(\frac{a}{2}\right)^{p} \max _{\mathbf{v} \in D}\|\mathbf{v}\|^{g}
$$

Легко видеть, что для всех $\mathbf{v} \in D$ выполняются неравенства

$$
s a \leqslant\|\mathbf{v}\| \leqslant \sqrt{m}(s+1) a .
$$

Следовательно, если $g \geqslant 0$, то

$$
\max _{\mathbf{v} \in D}\|\mathbf{v}\|^{g} \leqslant(\sqrt{m}(s+1) a)^{g} \leqslant\left(\frac{\sqrt{m}(s+1)}{s}\right)^{g}\|\mathbf{v}\|^{g} .
$$

Если $g<0$, то

$$
\max _{\mathbf{v} \in D}\|\mathbf{v}\|^{g} \leqslant \frac{1}{(s a)^{|g|}} \leqslant \frac{1}{\|\mathbf{v}\|^{|g|}}\left(\frac{\|\mathbf{v}\|}{s a}\right)^{|g|} \leqslant\left(\frac{\sqrt{m}(s+1)}{s}\right)^{|g|}\|\mathbf{v}\|^{g} .
$$


Значит, при любом $g$ получаем

$$
\max _{\mathbf{v} \in D}\|\mathbf{v}\|^{g} \leqslant c_{s}\|\mathbf{v}\|^{g}, \quad c_{s}=\left(\frac{\sqrt{m}(s+1)}{s}\right)^{|g|} .
$$

Выберем $s$ таким образом, чтобы выполнялось условие $s>d$. Тогда

$$
\gamma \equiv \frac{d}{s}<1
$$

Объединяя (17), (18) и (19), находим

$$
\begin{gathered}
\left|F(\mathbf{v})-F_{p}(\mathbf{v})\right| \leqslant c_{2} \gamma^{p}\|\mathbf{v}\|^{g}, \quad \mathbf{v} \in D \\
c_{2}=c c_{s} \max _{p} \frac{(p+1)^{m-1}}{2^{p}}
\end{gathered}
$$

Далее, очевидно,

$$
\Pi_{h} \subset \bigcup_{k} \mathscr{R}\left(a_{k}\right), \quad a_{k}=\left(1+\frac{1}{s}\right)^{k} \frac{h}{s}, \quad k=0,1, \ldots
$$

При этом $\Pi_{h}$ покрывается конечным числом множеств $\mathscr{R}\left(a_{k}\right)$, подчиненных условию $a_{k} \leqslant 1$. Следовательно, можно считать, что

$$
k \leqslant \frac{\log _{2} s+\log _{2} h^{-1}}{\log _{2}(1+1 / s)} .
$$

Чтобы завершить доказательство, достаточно заметить, что каждое из множеств $\mathscr{R}\left(a_{k}\right)$ содержит $(2 s+2)^{m}-(2 s)^{m}$ кубов со стороной $a_{k}$.

Теорема 2. Предположсим, что матрица $A$ порядка $n$ имеет әлементь

$$
a_{i j}=F\left(\mathbf{x}^{i}-\mathbf{y}^{j}\right)
$$

әде $F$ - полная асимптотически гладкая функиия, $а\left\{\mathbf{x}^{i}\right\} u\left\{\mathbf{y}^{j}\right\}-$ сетки вида (7) в т-мерном пространстве.

Пусть $0<h<1$. Для $1 \leqslant l \leqslant m$ обозначим через $\tau_{l}$ количество различных чисел вида $\theta^{l}(\mu, \nu)=s_{\mu}^{l}-t_{\nu}^{l}$ таких, что

$$
-h<\theta^{l}(\mu, \nu)<h, \quad 1 \leqslant \mu, \nu \leqslant n_{l},
$$

и положсим

$$
\tau=\tau_{1} \cdots \tau_{m}
$$

Пусть $0<\gamma<1$. Тогда для любого иелого $p \geqslant 0$ матрица А имеет тензорную аппроксимачию $A_{r}$ вида (1) со следующими оченками:

$$
\begin{gathered}
r \leqslant\left(c_{0}+c_{1} \log h^{-1}\right) p^{m-1}+\tau, \\
\left|\left\{A-A_{r}\right\}_{i j}\right| \leqslant c_{2} \gamma^{p}\left\|\mathbf{x}^{i}-\mathbf{y}^{j}\right\|^{g}, \quad 1 \leqslant i, j \leqslant n,
\end{gathered}
$$

әде константы $c_{0}, c_{1}, c_{2}$ зависят от $\gamma$ и $m$, но не зависят от $p$. 
ДокАЗАТЕЛЬство. Согласно лемме 1 достаточно установить существование сепарабельных аппроксимаций $F_{r} \approx F$ на множестве $\Pi_{h_{0}}$.

Теорема 1 утверждает, что сепарабельные аппроксимации сушествуют на $\Pi_{h}$. Если $h=h_{0}$, то все доказано (в этом случае $\tau=0$ ). В обшем случае следует рассмотреть сепарабельные функции теоремы 1 и увеличить соответствующее число членов $r$ таким образом, чтобы аппроксимации имели место на множестве $\Pi_{h} \cup \mathscr{M}_{h}$, где

$$
\mathscr{M}_{h}=\left\{\mathbf{v}: \mathbf{v}=\mathbf{x}^{i}-\mathbf{y}^{j} \in \Pi_{h_{0}} \backslash \Pi_{h}\right\} .
$$

Нетрудно проверить, что для этого достаточно добавить $\tau$ членов.

Заметим, что если сетки $\left\{\mathbf{x}^{i}\right\}$ и $\left\{\mathbf{y}^{j}\right\}$ имеют общие узлы, то, очевидно, $h_{0}=0$. В этом случае $\tau>0$. Если $\mathbf{x}^{i}=\mathbf{y}^{i}$, а $h$ определяется равенством

$$
h=\min _{1 \leqslant l \leqslant m} \min _{\substack{1 \leqslant \mu, \nu \leqslant n_{l} \\ \mu \neq \nu}}\left|s_{\mu}^{l}-s_{\nu}^{l}\right|,
$$

то, как легко видеть, $\tau=1$.

\section{§4. Виртуальные уровни}

В случае сеток в $m$-мерном пространстве естественно рассматривать тензорные аппроксимации с числом уровней $m$ ( $m$-членные прямые произведения). Однако интересно изучить также возможность введения виртуальных (не обусловленных геометрией задачи) уровней.

Пусть $m$ обозначает, по-прежнему, размерность пространства и матрица $A$ является $m$-уровневой с уровнями порядков $n_{1}, \ldots, n_{m}$.

Предположим, что $l$-й уровень разбивается на $\mu^{l}$ подуровней порядков $n_{1}^{l}, \ldots$, $n_{\mu l}^{l}$. Следовательно,

$$
n_{l}=n_{1}^{l} \cdots n_{\mu^{l}}^{l}, \quad 1 \leqslant l \leqslant m .
$$

Тогда $A$ можно рассматривать также как матрицу с числом уровней

$$
m^{\prime}=\sum_{l=1}^{m} \mu^{l}
$$

и поря дками уровней $n_{1}^{1}, \ldots, n_{\mu^{1}}^{1}, \ldots, n_{1}^{m}, \ldots, n_{\mu^{m}}^{m}$. Элементы такой $m^{\prime}$-уровневой матрицы удобно позиционировать с помощью составных мультииндексов

$$
\begin{gathered}
i \leftrightarrow \mathbf{i}=\left(\mathbf{i}^{1}, \ldots, \mathbf{i}^{m}\right), \quad j \leftrightarrow \mathbf{j}=\left(\mathbf{j}^{1}, \ldots, \mathbf{j}^{m}\right) \\
\mathbf{i}^{l}=\left(i_{1}^{l}, \ldots, i_{\mu^{l}}^{l}\right), \quad \mathbf{j}^{l}=\left(j_{1}^{l}, \ldots, j_{\mu^{l}}^{l}\right), \quad 1 \leqslant l \leqslant m .
\end{gathered}
$$

Полагаем, по-прежнему, что $A$ имеет элементы $a_{i j}=F\left(\mathbf{x}^{i}-\mathbf{y}^{j}\right)$. При этом в случае $m^{\prime}>m$ нам понадобятся некоторые дополнительные предположения относительно сеток $\left\{\mathbf{x}^{i}\right\}$ и $\left\{\mathbf{y}^{j}\right\}$. Будем считать, что каждая из одномерных сеток $\left\{s_{u}^{l}\right\}$, $\left\{t_{v}^{l}\right\}, 1 \leqslant u, v \leqslant n_{l}$, имеет вложенную периодическую структуру, означающую, что при естественном соответствии индексов и мультииндексов

$$
u \leftrightarrow \mathbf{u}=\left(u_{1}, \ldots, u_{\mu^{l}}\right), \quad v \leftrightarrow \mathbf{v}=\left(v_{1}, \ldots, v_{\mu^{l}}\right)
$$


многоуровневые матрицы

$$
S^{l}=\left[s_{\mathbf{u}}^{l}-s_{\mathbf{v}}^{l}\right], \quad T^{l}=\left[t_{\mathbf{u}}^{l}-t_{\mathbf{v}}^{l}\right]
$$

являются $\mu^{l}$-уровневыми матрицами, в которых все уровни, кроме последнего, имеют тёплицев тип (см. [2]). Другими словами, это блочно-тёплицевы матрицы, в которых блоки являются блочно-тёплицевыми матрицами и т. д., при этом на предпоследнем уровне имеем блочно-тёплицевы матрицы с блоками общего вида. Данное требование к сеткам выполняется, в частности, в случае равномерных сеток. Предположение относительно одномерных сеток приводит к следующим соотношениям:

$$
\begin{gathered}
s_{\mathbf{u}}^{l}=\zeta_{u_{1}}^{1}+\zeta_{u_{2}}^{2} \frac{1}{n_{1}^{l}}+\cdots+\zeta_{\mu^{l}-1}^{\mu^{l}-1} \frac{1}{n_{1}^{l} n_{2}^{l} \cdots n_{\mu^{l}-2}^{l}}+\zeta_{\mu^{l}}^{\mu^{l}} \frac{1}{n_{1}^{l} n_{2}^{l} \cdots n_{\mu^{l}-1}^{l}} \\
t_{\mathbf{v}}^{l}=\eta_{v_{1}}^{1}+\eta_{v_{2}}^{2} \frac{1}{n_{1}^{l}}+\cdots+\eta_{v_{\mu}{ }^{l}-1}^{\mu^{l}-1} \frac{1}{n_{1}^{l} n_{2}^{l} \cdots n_{\mu^{l}-2}^{l}}+\eta_{v_{\mu} l}^{\mu^{l}} \frac{1}{n_{1}^{l} n_{2}^{l} \cdots n_{\mu^{l}-1}^{l}} \\
0 \leqslant \zeta_{u^{l}}^{\mu^{l}} \leqslant 1, \quad 0 \leqslant \eta_{\mu_{\mu} \mu^{l}}^{\mu^{l}} \leqslant 1, \\
\zeta_{u_{k}}^{k}=\frac{u_{k}-1}{n_{k}^{l}}, \quad \eta_{v_{k}}^{k}=\frac{v_{k}-1}{n_{k}^{l}}, \quad 1 \leqslant u_{k}, v_{k} \leqslant n_{k}^{l}, \quad 1 \leqslant k \leqslant \mu^{l}-1 .
\end{gathered}
$$

Таким образом, можно ввести дополнительные переменные $\zeta^{k l}, \eta^{k l}$, удовлетворяющие условиям

$$
0 \leqslant \zeta^{k l}, \eta^{k l} \leqslant 1, \quad 1 \leqslant k \leqslant \mu^{l}
$$

и принимающие значения на определенных выше сетках $\left\{\zeta_{u_{k}}^{k}\right\},\left\{\eta_{v_{k}}^{k}\right\}$. Чтобы подчеркнуть зависимость этих сеток от номера уровня $l$, будем писать также

$$
\zeta_{u_{k}}^{k}=\zeta_{u_{k}}^{k l}, \quad \eta_{v_{k}}^{k}=\eta_{v_{k}}^{k l}
$$

При сделанных предположениях для $A$ можно рассмотреть тензорные аппроксимации вида

$$
A_{r}=\sum_{k=1}^{r}\left(A_{k 1}^{1} \times \cdots \times A_{k 1}^{\mu^{1}}\right) \times \cdots \times\left(A_{k m}^{1} \times \cdots \times A_{k m}^{\mu^{m}}\right)
$$

где матрица $A_{k l}^{j}$ имеет порядок $n_{j}^{l}, 1 \leqslant j \leqslant \mu^{l}, 1 \leqslant l \leqslant m$.

Обозначим через $n_{\min }$ минимальный порядок подуровней:

$$
n_{\min }=\min _{1 \leqslant l \leqslant m} \min _{1 \leqslant k \leqslant \mu^{l}} n_{k}^{l}
$$

ТЕОРема 3. Предположим, что т-уровневая матрица А порядка п имеет әлементи

$$
a_{i j}=F\left(\mathbf{x}^{i}-\mathbf{y}^{j}\right)
$$

әде $F$ - полная асимптотически гладкая функиия, $а\left\{\mathbf{x}^{i}\right\}$ u $\left\{\mathbf{y}^{j}\right\}-$ сетки вида (7) в т-мерном пространстве, и при этом l-й уровень разбивается на $\mu^{l}$ подуровней, $1 \leqslant l \leqslant m$, и соответствующие одномерные сетки удовлетворяют 
соотношениям вложенной периодичности (25) с общим числом подуровней $m^{\prime} \geqslant m$. Пусть

$$
\delta=\frac{1}{n_{\min }-1},
$$

где $n_{\min }-$ минимальный порядок подуровней (27).

Пусть $\delta<h<1$. Для $1 \leqslant l \leqslant m$ обозначим через $\tau_{l}$ количество различных чисел вида $\theta^{l}(\alpha, \beta)=s_{\alpha}^{l}-t_{\beta}^{l}$ таких, что

$$
-h-\delta<\theta^{l}(\alpha, \beta)<h+\delta, \quad 1 \leqslant \alpha, \beta \leqslant n_{l},
$$

u полоэимм

$$
\tau=\tau_{1}^{\mu^{1}} \cdots \tau_{m}^{\mu^{m}}
$$

Пусть $0<\gamma<1$.

Тогда для любого иелого $p \geqslant 0$ матрица $A$ имеет тензорную аппроксимацию $A_{r}$ вида (26) со следующими оценками:

$$
\begin{gathered}
r \leqslant\left(c_{0}+c_{1} \log h^{-1}\right) p^{m^{\prime}-1}+\tau \\
\left|\left\{A-A_{r}\right\}_{i j}\right| \leqslant c_{2} \gamma^{p}\left\|\mathbf{x}^{i}-\mathbf{y}^{j}\right\|^{g}, \quad 1 \leqslant i, j \leqslant n
\end{gathered}
$$

әде константы $c_{0}, c_{1}, c_{2}$ зависят от $\gamma$ и $m$, но не зависят от $p$.

ДокАЗАтЕльство. Будем рассматривать $F\left(v_{1}, \ldots, v_{m}\right)$ как функцию от $m^{\prime}$ скалярных переменных

$$
F\left(v_{1}, \ldots, v_{m}\right)=\mathscr{F}\left(v_{1}^{1}, \ldots, v_{\mu^{1}}^{1}, \ldots, v_{1}^{m}, \ldots, v_{\mu^{m}}^{m}\right),
$$

где

$$
v_{l}=v_{1}^{l}+v_{2}^{l} \frac{1}{n_{1}^{l}}+\cdots+v_{\mu^{l}-1}^{l} \frac{1}{n_{1}^{l} n_{2}^{l} \cdots n_{\mu^{l}-2}^{l}}+v_{\mu^{l}}^{l} \frac{1}{n_{1}^{l} n_{2}^{l} \cdots n_{\mu^{l}-1}^{l}} .
$$

Для краткости будем писать

$$
\mathscr{F}=\mathscr{F}\left(\mathbf{v}^{\prime}\right), \quad \mathbf{v}^{\prime}=\left(v_{1}^{1}, \ldots, v_{\mu^{1}}^{1}, \ldots, v_{1}^{m}, \ldots, v_{\mu^{m}}^{m}\right)
$$

В силу формул (25) достаточно считать, что

$$
-1 \leqslant v_{\nu}^{l} \leqslant 1, \quad 1 \leqslant \nu \leqslant \mu^{l}, \quad 1 \leqslant l \leqslant m .
$$

При этом получаем

$$
-1-\delta \leqslant v_{l} \leqslant 1+\delta, \quad 1 \leqslant l \leqslant m .
$$

Очевидно, теорема будет доказана, если мы найдем для $\mathscr{F}$ сепарабельную аппроксимацию

$$
\mathscr{F}_{r}\left(v_{1}^{1}, \ldots, v_{\mu^{1}}^{1}, \ldots, v_{1}^{m}, \ldots, v_{\mu^{m}}^{m}\right)=\sum_{k=1}^{r} \prod_{l=1}^{m} \prod_{\nu=1}^{\mu^{l}} \varphi_{\nu}^{k l}\left(v_{\nu}^{l}\right)
$$


с оценками $(29)$ и $(30)$ для $r$ и погрешности $\left|\mathscr{F}-\mathscr{F}_{r}\right|$ в области

$$
v_{1}^{l} \in[-1,1] \backslash(-h, h), \quad v_{\nu}^{l} \in[-1,1], \quad 2 \leqslant \nu \leqslant \mu^{l}, \quad 1 \leqslant l \leqslant m .
$$

Ясно, что

$$
\left(v_{1}^{l}, \ldots, v_{m}^{l}\right) \in \Pi_{h}=[-1,1]^{m} \backslash(-h, h)^{m} .
$$

Как и в доказательстве теоремы 1 , покроем $\Pi_{h}$ тем же семейством кубов, рассмотрим любой куб $D \subset \mathscr{R}(a)$ и разложим $F\left(v_{1}, \ldots, v_{m}\right)$ в ряд Тейлора в центральной точке $\mathbf{v}^{0}=\left(v_{1}^{0}, \ldots, v_{m}^{0}\right)$ куба $D$ :

$$
\begin{aligned}
& \mathscr{F}\left(v_{1}^{1}, \ldots, v_{\mu^{m}}^{m}\right) \approx \mathscr{F}_{p}\left(v_{1}^{1}, \ldots, v_{\mu^{m}}^{m}\right) \\
& \quad=\left.\sum_{k=0}^{p-1} \frac{1}{k !}\left(\Delta v_{1} \frac{\partial}{\partial \xi_{1}}+\cdots+\Delta v_{m} \frac{\partial}{\partial \xi_{m}}\right)^{k} F\left(\xi_{1}, \ldots, \xi_{m}\right)\right|_{\xi_{1}=v_{1}^{0}, \ldots, \xi_{m}=v_{m}^{0}} .
\end{aligned}
$$

В отличие от прежнего разложения (16) теперь будем рассматривать приращения следуюшего вида:

$$
\Delta v_{l}=\left(v_{1}^{l}-v_{l}^{0}\right)+v_{2}^{l} \frac{1}{n_{1}^{l}}+\cdots+v_{\mu^{l}-1}^{l} \frac{1}{n_{1}^{l} n_{2}^{l} \cdots n_{\mu^{l}-2}^{l}}+v_{\mu^{l}}^{l} \frac{1}{n_{1}^{l} n_{2}^{l} \cdots n_{\mu^{l}-1}^{l}}
$$

Согласно (36) и (28)

$$
\left|\Delta v^{l}\right| \leqslant \frac{a}{2}+\delta
$$

Обозначим через $D_{\delta}$ куб со стороной $a+2 \delta$ и с тем же центром, что и у куба $D$. Кроме того, рассмотрим $m^{\prime}$-мерную область

$$
D^{\prime}=\left\{\mathbf{v}^{\prime}:\left(v_{1}^{1}, \ldots, v_{1}^{l}\right) \in D, v_{\nu}^{l} \in[-1,1], 1 \leqslant \nu \leqslant \mu^{l}, 1 \leqslant l \leqslant m\right\} .
$$

Если $\mathbf{v}^{\prime} \in D^{\prime}$, то $\mathbf{v}=\left(v_{1}, \ldots, v_{m}\right) \in D_{\delta}$. Поэтому оценка (17) модифицируется таким образом:

$$
\left|\mathscr{F}\left(\mathbf{v}^{\prime}\right)-\mathscr{F}_{p}\left(\mathbf{v}^{\prime}\right)\right| \leqslant(p+1)^{m-1} c \frac{d^{p}}{(s a-\delta)^{p}}\left(\frac{a}{2}+\delta\right)^{p} \max _{\mathbf{v} \in D_{\delta}}\|\mathbf{v}\|^{g} .
$$

В данном случае

$$
s a-\delta \leqslant\|\mathbf{v}\| \leqslant \sqrt{m}((s+1) a+\delta) .
$$

Отсюда

$$
\begin{gathered}
\max _{\mathbf{v} \in D_{\delta}}\|\mathbf{v}\|^{g} \leqslant c_{s}(a, \delta)\|\mathbf{v}\|^{g}, \quad \mathbf{v} \in D_{\delta} \\
c_{s}(a, \delta)=\left(\frac{\sqrt{m}((s+1) a+\delta)}{s a-\delta}\right)^{|g|} .
\end{gathered}
$$

Как и раньше, выберем $s>d$ и положим

$$
\gamma \equiv \frac{d}{s}<1
$$


Получаем

$$
\left|\mathscr{F}\left(\mathbf{v}^{\prime}\right)-\mathscr{F}_{p}\left(\mathbf{v}^{\prime}\right)\right| \leqslant c \frac{(p+1)^{m-1}}{2^{p}}\left(\frac{s(a+2 \delta)}{s a-\delta}\right)^{p} c_{s}(a, \delta) \gamma^{p}\|\mathbf{v}\|^{g}
$$

Неравенство (30) с константой $c_{2}$, не зависящей от $p$, является следствием предельных соотношений

$$
\lim _{\delta \rightarrow 0} c_{s}(a, \delta)=c_{s},
$$

где $c_{s}$ определяется согласно (18),

$$
\lim _{\delta \rightarrow 0}\left(\frac{s(a+2 \delta)}{s a-\delta}\right)^{p}=1, \quad \lim _{p \rightarrow \infty} \frac{(p+1)^{m-1}}{2^{p}}=0 .
$$

Оценка для $r$ получается в полной аналогии с соответствующей оценкой теоремы 1 и последующим ее уточнением в теореме 2.

\section{§5. Численные примеры}

Рассмотрим равномерную сетку на единичном кубе $[0,1]^{3}$ с числом узлов по каждой оси $N$ и трехуровневые матрицы $A$ с порядками уровней $n_{1}=n_{2}=n_{3}=$ $N$. Пусть $N=10$. Тогда порядок матрицы $A$ равен $n=N^{3}=1000$.

Будем считать, что элементы матрицы $A$ имеют вид $(3),(4)$ и соответствующая функция $F(\mathbf{v})$ зависит только от $\rho=\|\mathbf{v}\|$.

Чтобы построить для $A$ тензорные аппроксимации, применим алгоритм сингулярного разложения для соответствующей матрицы $A_{3}(\S 2)$. При этом мы получим тензорную аппроксимацию вида

$$
A \approx A_{p}=\sum_{k=1}^{p} B^{k} \times W^{k}
$$

где $B^{k}$ и $W^{k}$ - матрицы порядка $N^{2}$ и $N$ соответственно. Затем мы опять применяем алгоритм сингулярного разложения для матриц $\left(B^{k}\right)_{2}$ (отображение $B^{k} \rightarrow$ $\left(B^{k}\right)_{2}$ определяется так же, как $A \rightarrow A_{2} ;$ см. $\left.\S 2\right)$ и получаем тензорные аппроксимации вида

$$
B^{k} \approx \sum_{j=1}^{r_{k}} U_{j}^{k} \times V_{j}^{k}
$$

где $U_{j}^{k}$ и $V_{j}^{k}$ - матрицы порядка $N$. В результате матрица $A$ аппроксимируется суммой

$$
r=r_{1}+\cdots+r_{p}
$$

прямых произведений вида $U_{j}^{k} \times V_{j}^{k} \times W^{k}$.

При применении алгоритма сингулярного разложения выбирается аппроксимация наименьшего ранга, обеспечивающая заданную относительную точность $\varepsilon$ по норме Фробениуса. 
ТАБлицА 1. Тензорные аптроксимации для функции $f=1 / \rho$

\begin{tabular}{|c|c|c|c|c|}
\hline$\varepsilon$ & $10^{-4}$ & $10^{-5}$ & $10^{-6}$ & $10^{-7}$ \\
\hline$r$ & 37 & 49 & 62 & 77 \\
\hline$r_{j}$ & $6,6,6,6,7,6$ & $6,7,7,7,7,8,7$ & $7,7,7,7,8,9,9,8$ & $8,8,8,8,9,9,9,9,9$ \\
\hline
\end{tabular}

ТАБлицА 2. Тензорные аппроксимации для функции $f=1 / \rho^{2}$

\begin{tabular}{|c|c|c|c|c|}
\hline$\varepsilon$ & $10^{-4}$ & $10^{-5}$ & $10^{-6}$ & $10^{-7}$ \\
\hline$r$ & 36 & 48 & 63 & 76 \\
\hline$r_{j}$ & $6,5,6,6,7,6$ & $7,6,6,7,7,8,7$ & $7,7,8,8,8,8,9,8$ & $8,8,8,8,8,9,9,9,9$ \\
\hline
\end{tabular}

В таблицах 1, 2 приводятся примеры для двух различных функций $F=F(\rho)$, удовлетворяющих требованию полной асимптотической гладкости.

Заметим, что каждое из прямых произведений состоит из трех матриц порядка 10. Таким образом, в случае 37 членов для запоминания тензорной аппроксимации достаточно хранить $37 \cdot 3 \cdot 100=11100$ чисел. В действительности, поскольку матрищы $W^{k}$ во многих членах одинаковы, памяти нужно почти на одну треть меньше: $37 \cdot 2 \cdot 100+6 \cdot 100=8000$. Для хранения исходной матрицы требуется $1000^{2}=1000000$ чисел.

\section{Список литературы}

1. Воеводин В. В. Об одном методе понижения порядка матриц при решении интегралњншх уравнений // Численный анализ на ФОРТРАНе. М.: Изд-во МГУ, 1979. С. 21-26.

2. Воеводин B. B., Тыртышников E. E. Вычислителшные процессы с тёплицевыми матрицами. М.: Наука, 1987.

3. Hackbusch W., Nowak Z.P. On the fast matrix multiplication in the boundary element method by panel clustering // Numer. Math. 1989. V. 54. № 4. P. 463-491.

4. Myagchilov M. V., Tyrtyshnikov E. E. A fast matrix-vector multiplier in discrete vortex method // Russian J. Numer. Anal. Math. Modelling. 1992. V. 7. № 4. P. 325-342.

5. Rokhlin V. Rapid solution of integral equations of classical potential theory // J. Comput. Phys. 1985. V. 60. P. 187-207.

6. Rokhlin $V$. Rapid solution of integral equations of scattering theory in two dimensions // J. Comput. Phys. 1990. V. 86. P. 414-439.

7. Тыртышииков E. E. Методы быстрого умножения и решение уравнений // Матричные методы и вычисления. М.: ИВМ РАН, 1999. С. 4-41.

8. Tyrtyshnikov E. E. Mosaic ranks and skeletons // Numerical analysis and its applications. Proceedings of WNAA-96. Berlin: Springer-Verlag, 1996. P. 505-516. (Lecture Notes in Comput. Sci. V. 1196.)

9. Tyrtyshnikov E. E. Mosaic-skeleton approximations // Calcolo. 1996. V. 33. № 1-2. P. 47-57.

10. Горейнов C.A. Мозаично-скелетонные аппроксимации матриц, порожденных асимптотически гладкими и осцилляционными ядрами // Матричные методы и вычисления. М.: ИВМ РАН, 1999. С. 42-76.

11. Sun X., Pitsianis N. P. A matrix version of the fast multipole method // SIAM Rev. 2001. V. 43. № 2. P. 289-300. 
12. Горейнов С.А., Замарашкин Н. Л., Тьртыиников Е. Е. Псевдоскелетные аппроксимации матриц // Докл. РАН. 1995. Т. 343. № 2. С. 151-152.

13. Goreinov S. A., Tyrtyshnikov E. E. The maximal-volume concept in approximation by low-rank matrices // Contemp. Math. 2001. V. 208. P. 47-51.

14. Goreinov S. A., Tyrtyshnikov E. E., Yeremin A. Y. Matrix-free iterative solution strategies for large dense linear systems // Numer. Linear Algebra Appl. 1997. V. 4. № 4 . P. 273-294.

15. Goreinov S. A., Tyrtyshnikov E. E., Zamarashkin N.L. A theory of pseudo skeleton approximations // Linear Algebra Appl. 1997. V. 261. P. 1-21.

16. Tyrtyshnikov E.E. Incomplete cross approximation in the mosaic-skeleton method // Computing. 2000. V. 64. № 4. P. 367-380.

17. Ibraghimov I. Application of 3-way decomposition for matrix compression // Numer. Linear Algebra Appl. 2002. V. 9. №6-7. P. 551-565.

18. Tyrtyshnikov E. E. Kronecker-product approximations for some function-related matrices // Linear Algebra Appl. (to appear).

19. Van Loan C.F., Pitsianis N.P. Approximation with Kronecker products // Linear algebra for large scale and real-time applications. Proc. of the NATO Advanced Study Institute, Leuven, Belgium, August 3-14, 1992. Dordrecht: Kluwer Acad. Publ., 1993. P. 293-314. (NATO Adv. Sci. Inst. Ser. E Appl. Sci. V. 232.)

Институт вычислительной математики РАН, г. Москва
Поступила в редакцию 31.10 .2002 